\title{
Direct fragmentation of quarkonia including Fermi motion using light-cone wave function
}

\author{
M.A. Gomshi Nobary, B. Javadi \\ Department of Physics, Faculty of Science, Razi University, Kermanshah, Iran
}

Published online: 6 June 2007 - C S Springer-Verlag / Società Italiana di Fisica 2007

In Fig. 1 of the paper as the confinement parameter $\beta$ increases the width of the wave packet should increase. Due to a mistake in the program which produces this figure, the procedure is shown in reverse order. The shape of the packet is also affected by this mistake. The correct behavior is shown in the following Fig. 1.

There were also some typing errors in the explicit form of our fragmentation functions which appear in the appendix. The correct form of the appendix is given below. All our results and other figures are correct. The correct form of the whole paper is also available at arXiv:hep-ph/0504098 v3 12 July 2006.
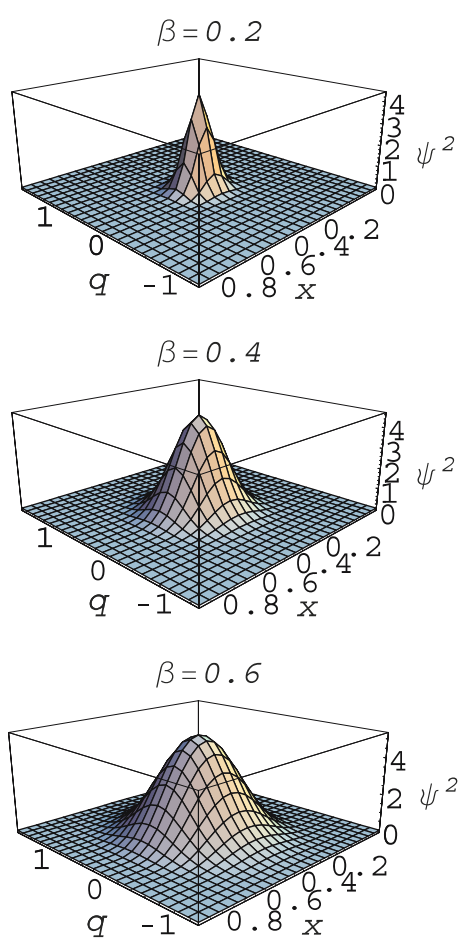

Fig. 1.

The online version of the original article can be found at http://dx.doi.org/10.1140/epjc/s2005-02266-1

\section{Appendix}

The explicit form of (28) and (29) for quarkonia cast into the following

$$
\begin{aligned}
D\left(z, \mu_{\circ}, \beta=0\right)= & \frac{\alpha_{\mathrm{s}}^{2} C_{\mathrm{F}}^{2}\left\langle k_{\mathrm{T}}^{\prime}{ }^{2}\right\rangle^{1 / 2}}{16 m \mathcal{F}} \\
\times & \left\{z ( 1 - z ) ^ { 2 } \left[\xi^{2} z^{4}+2 \xi z^{2}\left(4-4 z+5 z^{2}\right)\right.\right. \\
& \left.\left.\quad+\left(16-32 z+24 z^{2}-8 z^{3}+9 z^{4}\right)\right]\right\},
\end{aligned}
$$

and

$$
\begin{aligned}
D\left(z, \mu_{\circ}, \beta\right)= & \frac{\pi^{2} \alpha_{\mathrm{s}}^{2} C_{\mathrm{F}}^{2}\left\langle k_{\mathrm{T}}^{\prime 2}\right\rangle^{1 / 2}}{2 m} \\
& \times \int \frac{\mathrm{d} q \mathrm{~d} x q x^{2} z(1-z)^{2}\left|\psi_{\mathrm{M}}\right|^{2}}{\mathcal{G}} \mathcal{H},
\end{aligned}
$$

where $\mathcal{F}, \mathcal{G}$ and $\mathcal{H}$ read as

$$
\begin{aligned}
\mathcal{F}= & {\left[\xi^{2} z^{4}-(z-2)^{2}(3 z-4)+\xi z^{2}\left(8-7 z+z^{2}\right)\right]^{2}, } \\
\mathcal{G}=\{ & \left\{\left[(1-z)^{2}+\xi x^{2} z^{2}+(1-(1-x) z)^{2}\right]\right. \\
& \left.\times\left[\eta(-1+z)+\xi(-1+x) x z^{2}-1+\left(1-x+x^{2}\right) z\right]\right\}^{2},
\end{aligned}
$$

and

$$
\begin{aligned}
\mathcal{H}=\{ & 1-4(1-x) z+2\left(4-10 x+7 x^{2}\right) z^{2} \\
& +4\left(-1+4 x-5 x^{2}+x^{3}\right) z^{3} \\
& +\left(1-4 x+8 x^{2}-4 x^{3}+x^{4}\right) z^{4} \\
& +\eta \xi z^{2}\left[1-2 x+z^{2}+x^{2}\left(2-2 z+z^{2}\right)\right] \\
& +\eta\left(2+(4 x-6) z+\left(9-8 x+2 x^{2}\right) z^{2}\right. \\
& \left.\quad-2\left(2-x+x^{2}\right) z^{3}+\left(1+x^{2}\right) z^{4}\right) \\
& +\xi z^{2}\left(1+2 x^{3}(2-3 z) z+z^{2}+2 x^{4} z^{2}\right. \\
& \left.\quad+x\left(-2+2 z-4 z^{2}\right)+x^{2}\left(2-8 z+9 z^{2}\right)\right) \\
& \left.+\eta^{2}(1-z)^{2}+\xi^{2}(1-x)^{2} x^{2} z^{4}\right\} .
\end{aligned}
$$

Here $\xi=\left\langle k_{\mathrm{T}}^{\prime 2}\right\rangle / m^{2}$ and $\eta=q^{2} / m^{2}$. $A_{\mathrm{M}}$ is calculated using the normalisation condition of the wave function, e.g. equation (3). 